\title{
Stress associated median canaliform dystrophy of Heller in medical students more prominent on dominant thumb nail: A case series
}

\author{
Annie Raizada, Maitreyee Panda, Debasmita Behera, Chinmoy Raj
}

\author{
Department of Dermatology, Venereology and Leprosy, IMS \& SUM Hospital, SOA Deemed to be University, \\ Kalinga Nagar - 751 003, Bhubaneswar, Odisha, India
}

Corresponding author: Dr. Annie Raizada, E-mail: raizada.annie001@gmail.com

\begin{abstract}
Median canaliform dystrophy of Heller is an unusual condition, presenting morphologically as a central or paramedian longitudinal groove with lateral projections, creating an appearance of 'inverted fir tree- like' pattern. Exact etiopathogenesis is unclear, however it has been proposed that Median Canaliform Dystrophy of Heller, is an acquired entity. Repetitive nail plate and cuticle trauma is a common association and treatment with oral retinoids have been hypothesized as the cause, however the etiology remains elusive for most affected individuals. Treatment is often unsatisfactory and prolonged, however topical $0.1 \%$ tacrolimus, $0.05 \%$ tazarotene and injectable triamcinolone acetonide have been tried with notable improvement. Psychiatric consultation should be sought when associated with obsessive-compulsive, impulse control or depressive disorders. We present a case series of three medical students, with stress associated Median Canaliform Dystrophy of Heller, typically more severe on right thumb nail in all three cases.
\end{abstract}

Key words: Stress; Medical student; Median nail dystrophy; Dominant thumb

\section{INTRODUCTION}

Median Canaliform Dystrophy of Heller, is a rare nail abnormality, presenting clinically as central or paramedian groove or split with multiple, transverse parallel lines, typically involving one or both thumb nails, however other nails can also be involved. Heller, recorded the first ever case of this disorder in 1928 [1]. The diagnosis is mostly based on Clinical features. Exact etiopathogenesis is elusive, however self inflicted trauma is implicated in most cases. Majority of cases are acquired but few cases with familial occurrence and following use of oral retinoids have been reported [2].

Male:Female ratio is $1: 1$ [3] and mean age of occurrence is 25.72 years [4]. The closest differential diagnosis to this condition is Habit Tic Deformity. Multiple treatment modalities have been tried with unsatisfactory outcome.
We report a case series of Median Nail Dystrophy of Heller in three medical students, with a common background of underlying stress related to exams and lesions predominantly on the right thumb nail.

\section{CASE REPORTS}

\section{Case 1}

A 24 years old, intern presented with single longitudinal groove with multiple oblique ridges running in a paramedian and outward manner, giving a 'fir tree - like' appearance on bilateral thumb nails since three years. Lesions were more prominent on right thumb nail. Lunula was enlarged in size than normal on bilateral thumb nails (Fig. 1). The median longitudinal groove extended from proximal nail fold upto distal nail edge. Cuticle was normal in bilateral thumb nails, with mild periungual scaling. No other finger nails were affected. No associated skin or mucosal lesions were present.

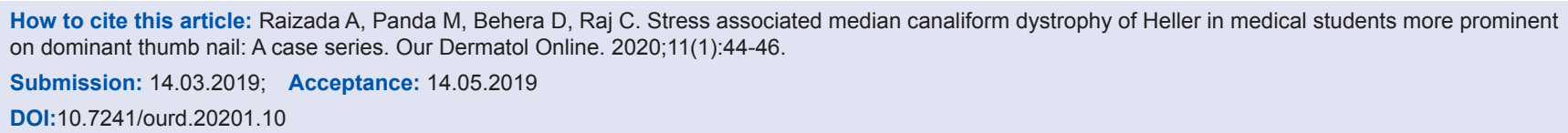




\section{Case 2}

A 22 years old, male final year M.B.B.S. student, presented with similar complaints as that described for case 1 with additional findings of periungual hypopigmentation with superficial scaling and soggy appearance (Fig. 2).

\section{Case 3}

The third patient was 21 years old male, third year M.B.B.S. student, presented with similar signs and symptoms (Fig. 3). All the patients visited us due to cosmetic concern regarding the nails. There was a common background of underlying stress related to exams. With every semester exams the habit of nail picking/biting got aggravated and severity of nail involvement increased. There was no history of oral retinoid or other medication use, no history of contact with irritants or allergens. There was no systemic involvement. All routine investigations were normal. Nail dermoscopy, imaging studies and subungual scraping for $\mathrm{KOH}$ was done to rule out any underlying nail pathology. A diagnosis of Median Canaliform Dystrophy of Heller was made based on clinical evaluation. Psychiatric referral was done.

\section{DISCUSSION}

Median Canaliform Dystrophy of Heller, also known as Dystrophia unguis mediana canaliformis, nevus striatus unguis or soleonychia, presents clinically as longitudinal central or paramedian groove, with multiple lateral transverse projections, giving a pattern of 'inverted fir tree - like' appearance. In severe cases, the nail plate can split along the groove. Other features like enlargement and redness of lunula, thickening of proximal nail fold can be found. There is no sex predilection and mean age of presentation is 25.72 years [4]. Involvement most commonly is symmetrical and frequently involves the thumb, however other fingers and toes may be affected. In our case series there was predominant involvement of right thumb. This can be explained hypothetically because of involvement of dominant hand with recurrent Koebner phenomenon and relative ease of self inflicting trauma.

It is an acquired entity however Sweeney et al has reported familial clustering of cases in 2005 [3]. Exact etiopathogenesis is not fully understood, however

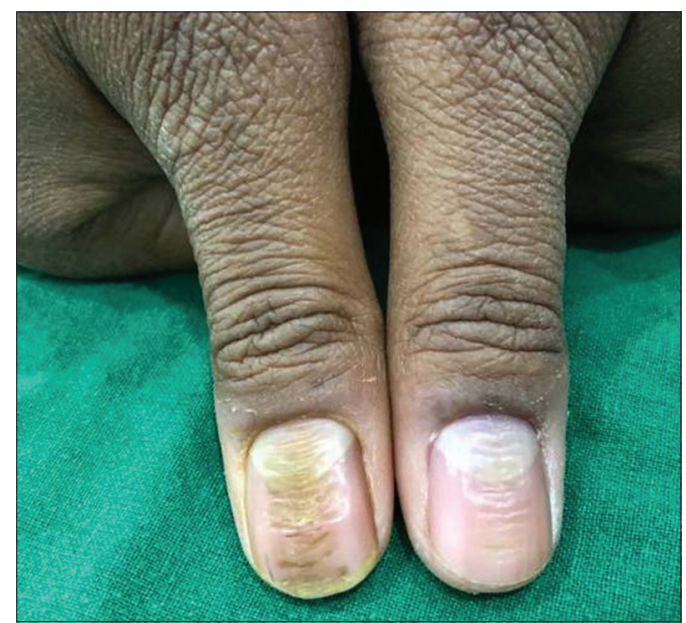

Figure 1: Single longitudinal groove with multiple oblique ridges running in paramedian and outward manner giving an 'inverted fir tree - like' appearance on both thumb nails, more prominent on right thumb nail in a 24 years male medical student. Size of Lunula enlarged on bilateral nails.

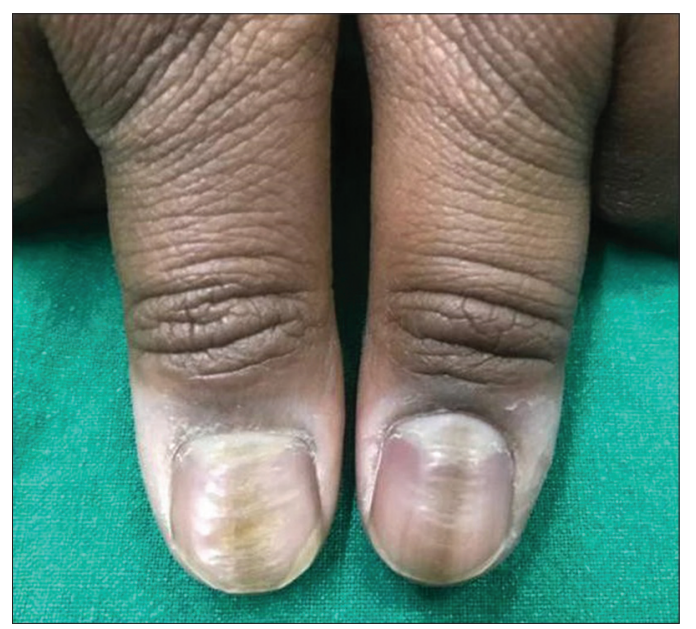

Figure 2: A single median longitudinal ridge with lateral projections, giving an 'inverted fir tree -like' pattern with enlarged lunula and periungual soggy appearance on both thumb nails, more prominent on right thumb nail, in a 22 years male medical student.

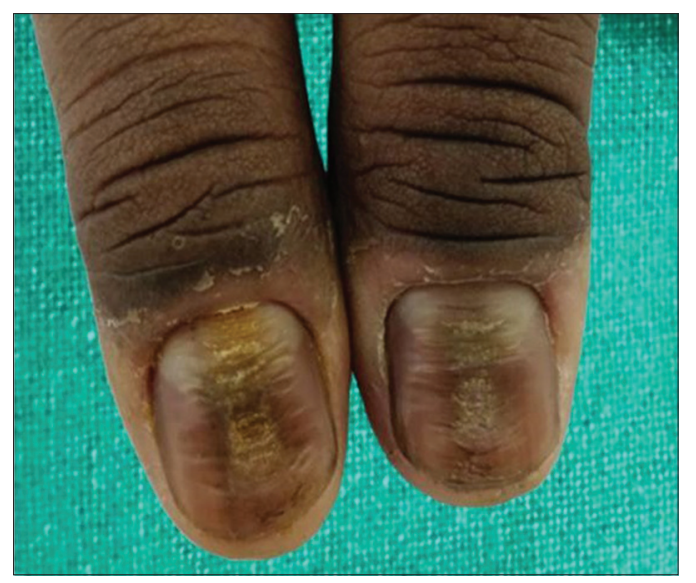

Figure 3: A single median longitudinal ridge with lateral projections, giving an 'inverted fir tree -like' pattern with enlarged lunula on both thumb nails more prominent on right thumb nail and bilateral mild superficial scaling, in a 21 years male medical student. 
conditions causing temporary defect in nail matrix due to focal infection, discretization, self inflicted trauma to nail or nail bed or repetitive pushing down of cuticle or proximal nail folds, use of oral retinoids, have been thought to be the causative factors. Reason for formation of longitudinal groove can be attributed to absence of keratinocytic adhesions within nail matrix with dyskeratosis with splitting of nail plate due to weaker tensile strength [4].

The most significant differential diagnosis of MCD of Heller is Habit Tic Deformity. Habit tic deformity is caused by habitual or constant rubbing of the thumb's proximal nail fold and cuticle by the tip of second digit, resulting in longitudinal nail defects. Themain differentiating feature is normal cuticle in MCD of Heller,while cuticle is affected or completely lost in Habit Tic Deformity [5].

Most cases are poorly responsive to treatment. Topical application of tacrolimus $(0.1 \%)$ and tazarotene $(0.05 \%)$ [6], have been tried with notable improvement and acts by normalizing the process of keratinization with additional anti-inflammatory role. Satisfactory results have been seen with intralesional steroid injection using triamcinolone acetonide. Psychiatry consultation is indicated in cases of obsessivecompulsive, anxiety, depression or impulse-control disorders. Non-medical therapy includes covering the nail plate with nail wrap or tape, to avoid direct trauma to the nails.

\section{CONCLUSION}

The novelty of this case series lies in the fact, that all the three reported patients had aggravation of symptoms with stress related to examinations, predominantly involving dominant thumb nails. This is possibly the first case series reported to the best of our knowledge.

\section{Consent}

The examination of the patient was conducted according to the Declaration of Helsinki principles.

\section{REFERENCES}

1. Beck M, Wilkinson S. Disorders of nails: Medican canaliform dystrophy. In: Burns T, Breathnach S, Cox N, Griffiths C, editors. Rook's Textbook of Dermatology. $7^{\text {th }}$ ed. Oxford: Blackwell Science. 2004. pp. 54-5.

2. Avhad G, Ghuge P. Median canaliform dystrophy of Heller. Indian Pediatr. 2013;50:1073.

3. Sweeney SA, Cohen PR, Schulze KE, Nelson BR. Familial Median Canaliform nail dystrophy. Cutis. 2005;75:161-5.

4. Kota R, Pilani A, Nair PA. Median nail dystrophy involving the thumb nail. Ind J Der. 2016;16:120.

5. Singal A, Daulatabad D. Nail tic disorders: Manifestations, pathogenesis and management. Ind J Dermatol Venereol Leprol. 2017;83:19-26.

6. Madke B, Gadkari R, Nayak C. Median canaliform dystrophy of Heller. Indian Dermatol Online J. 2012;3:224-5.

Copyright by Annie Raizada, et al. This is an open-access article distributed under the terms of the Creative Commons Attribution License, which permits unrestricted use, distribution, and reproduction in any medium, provided the original author and source are credited.

Source of Support: Nil, Conflict of Interest: None declared. 